J. Nonlinear Var. Anal. 3 (2019), No. 2, pp. 171-179

Available online at http://jnva.biemdas.com

https://doi.org/10.23952/jnva.3.2019.2.06

\title{
A FORWARD-BACKWARD ALGORITHM FOR THE DC PROGRAMMING IN HILBERT SPACES
}

\author{
CHIH-SHENG CHUANG \\ Department of Applied Mathematics, National Chiayi University, Chiayi, Taiwan \\ Dedicated to Professor Wataru Takahashi on the occasion of his 75th birthday
}

\begin{abstract}
In this paper, we introduce a forward-backward algorithm to solve the DC programming problem and establish convergence theorems in the framework of finite dimensional real Hilbert spaces. Our results can also be extended to the generalized DC programming.

Keywords. DC programming; Maximal monotone; Strongly convex; Semicontinuous and convex function.
\end{abstract}

2010 Mathematics Subject Classification. 49J53, 49M37, 90C26.

\section{INTRODUCTION}

The following nonconvex problem is called the DC programming:

$$
\text { (DCP) Find } \bar{x} \in \arg \min _{x \in \mathbb{R}^{n}}\{f(x)=g(x)-h(x)\},
$$

where $g, h: \mathbb{R}^{n} \rightarrow \mathbb{R}$ are proper, lower semicontinuous, and convex functions. Here, the function $f$ is called a DC function, and functions $g$ and $h$ are called DC components of $f$. (In DC programming, the convention $(+\infty)-(+\infty)=+\infty$ has been adopted to avoid the ambiguity $(+\infty)-(+\infty)$ that does not present any interest.) It is well known that a necessary condition for $x \in \operatorname{dom}(f):=\left\{x \in \mathbb{R}^{n}: f(x)<\infty\right\}$ to be a local minimizer of $f$ is $\partial h(x) \subseteq \partial g(x)$. But, this condition is hard to be reached. So, many researchers focus their attentions on finding points such that $\partial h(x) \cap \partial g(x) \neq \emptyset$, where $x$ is called a critical point of $f$ [14]. For more details about DC functions and DC programming, one refers to [7, 8 , $9,10,11,12,13,14,17,18,19]$.

In 2016, Souza, Oliveira, and Soubeyran [18] proposed a proximal linearized algorithm to study DC programming.

Algorithm 1.1. (Proximal linearized Algorithm [18]) Let $\left\{\beta_{n}\right\}_{n \in \mathbb{N}}$ be a sequence in $(0, \infty)$, and let $g, h: \mathbb{R}^{k} \rightarrow \mathbb{R}$ be proper lower semicontinuous and convex functions. Let $\left\{x_{n}\right\}_{n \in \mathbb{N}}$ be generated by the

E-mail address: cschuang@ mail.ncyu.edu.tw.

Received March 20, 2019; Accepted May 2, 2019.

(c)2019 Journal of Nonlinear and Variational Analysis 
following

$$
\left\{\begin{array}{l}
x_{1} \in H_{1} \text { is chosen arbitrarily, } \\
\text { Compute } w_{n} \in \partial h\left(x_{n}\right), \\
x_{n+1}:=\arg \min _{u \in H_{1}}\left\{g(u)+\frac{1}{2 \beta_{n}}\left\|u-x_{n}\right\|^{2}-\left\langle w_{n}, u-x_{n}\right\rangle\right\}, n \in \mathbb{N} . \\
\text { stop criteria: } x_{n+1}=x_{n} .
\end{array}\right.
$$

In 2008, Maingé and Moudafi [16] proposed the generalized DC programming, which is an extension of DC programming-not too large to allow us the use of the arsenal of powerful tools in convex analysis and convex optimization but sufficiently wide to cover most real-world nonconvex optimization problems.

$$
\left(\text { GDCP) } \operatorname{argmin}_{x \in \mathbb{R}^{k}}\{f(x)=\varphi(x)+g(x)-h(x)\},\right.
$$

where $\varphi: \mathbb{R}^{k} \rightarrow \mathbb{R}$ is a continuous differentiable function (not necessary convex) and $g, h: \mathbb{R}^{k} \rightarrow \mathbb{R} \cup$ $\{+\infty\}$ are proper, convex, and lower semicontinuous functions. Further, for problem (GDCP), let $\Omega_{\mathrm{GDCP}}$ be defined by

$$
\Omega_{\mathrm{GDCP}}:=\left\{x \in \mathbb{R}^{k}: \partial h(x) \cap[\partial g(x)+\nabla \varphi(x)] \neq \emptyset\right\},
$$

and the elements of $\Omega_{\mathrm{GDCP}}$ are also called critical points of the function $f(x)=\varphi(x)+g(x)-h(x)[1,6]$.

Besides, we know that (GDCP) is related to the following problem:

$$
\arg \min \{\varphi(x)+g(x): x \in H\}
$$

where $H$ is a real Hilbert space, and $\varphi: H \rightarrow(-\infty, \infty]$ and $g: H \rightarrow(-\infty, \infty]$ are proper, lower semicontinuous, and convex functions. From the literature, problem (1.1) has many important applications, including multiresolution sparse regularization, Fourier regularization, hard-constrained inconsistent feasibility, and alternating projection signal synthesis problems; see, e.g., [5, 15] and the references therein.

For problem (1.1), we observed that the following forward-backward algorithm (see, e.g., [2, Corollary 27.9])

$$
\left\{\begin{array}{l}
z_{n}:=\operatorname{prox}_{\beta, f}\left(x_{n}-\beta \nabla g\left(x_{n}\right)\right), \\
x_{n+1}:=x_{n}+k_{n}\left(z_{n}-x_{n}\right), n \in \mathbb{N},
\end{array}\right.
$$

where $\beta>0, f: H \rightarrow(-\infty, \infty]$ is a proper, lower semicontinuous, and convex function, $g: H \rightarrow(-\infty, \infty]$ is a proper, lower semicontinuous, convex, and Fréchet differentiable function, and $\operatorname{prox}_{\beta, f}$ is a proximal operator of $f$ with $\beta$.

Further, Beck and Teboulle [3] gave the following Fast Iterative Shrinkage-Thresholding Algorithm (FISTA).

$$
\left\{\begin{aligned}
z_{n+1} & :=\operatorname{prox}_{\beta, f}\left(x_{n}-\beta \nabla g\left(x_{n}\right)\right), \\
t_{n+1} & :=\frac{1+\sqrt{1+4 t_{n}^{2}}}{2} \\
x_{n+1} & :=z_{n+1}+\frac{t_{n}-1}{t_{n+1}}\left(z_{n+1}-z_{n}\right), n \in \mathbb{N} .
\end{aligned}\right.
$$

In this paper, motivated by the above works, we introduce a forward-backward algorithm to solve the DC programming problem and establish convergence theorems in the framework of finite dimensional Hilbert spaces. Our results can also be extended to the generalized DC programming. 


\section{PRELIMINARIES}

Let $H$ be a real Hilbert space with inner product $\langle\cdot, \cdot\rangle$ and norm $\|\cdot\|$. We denote the strong and weak convergence of $\left\{x_{n}\right\}_{n \in \mathbb{N}}$ to $x \in H$ by $x_{n} \rightarrow x$ and $x_{n} \rightarrow x$, respectively. For each $x, y, u, v \in H$, and $\lambda \in \mathbb{R}$, we have

$$
\begin{gathered}
\|x+y\|^{2}=\|x\|^{2}+2\langle x, y\rangle+\|y\|^{2}, \\
\|\lambda x+(1-\lambda) y\|^{2}=\lambda\|x\|^{2}+(1-\lambda)\|y\|^{2}-\lambda(1-\lambda)\|x-y\|^{2}, \\
2\langle x-y, u-v\rangle=\|x-v\|^{2}+\|y-u\|^{2}-\|x-u\|^{2}-\|y-v\|^{2} .
\end{gathered}
$$

Definition 2.1. Let $H$ be a real Hilbert space. Let $B: H \rightarrow H$ be a mapping, and let $\rho>0$. Then

(i) $B$ is monotone if $\langle x-y, B x-B y\rangle \geq 0$ for all $x, y \in H$.

(ii) $B$ is $\rho$-strongly monotone if $\langle x-y, B x-B y\rangle \geq \rho\|x-y\|^{2}$ for all $x, y \in H$.

Definition 2.2. Let $H$ be a real Hilbert space, and let $B: H \multimap H$ be a set-valued mapping with domain $\mathscr{D}(B):=\{x \in H: B(x) \neq \emptyset\}$. Then

(i) $B$ is monotone if $\langle u-v, x-y\rangle \geq 0$ for any $u \in B(x)$ and $v \in B(y)$.

(ii) $B$ is maximal monotone if its graph $\{(x, y): x \in \mathscr{D}(B), y \in B(x)\}$ is not properly contained in the graph of any other monotone mapping.

(iii) $B$ is $\rho$-strongly monotone $(\rho>0)$ if $\langle x-y, u-v\rangle \geq \rho\|x-y\|^{2}$ for all $x, y \in H$, and all $u \in B(x)$, and $v \in B(y)$.

Definition 2.3. Let $H$ be a real Hilbert space, and let $f: H \rightarrow(\infty, \infty]$ be a function. Then

(i) $f$ is proper if $\{x \in H: f(x)<\infty\} \neq \emptyset$.

(ii) $f$ is lower semicontinuous if $\{x \in H: f(x) \leq r\}$ is closed for each $r \in \mathbb{R}$.

(iii) $f$ is convex if $f(t x+(1-t) y) \leq t f(x)+(1-t) f(y)$ for every $x, y \in H$ and $t \in[0,1]$.

(iv) $f$ is $\rho$-strongly convex $(\rho>0)$ if

$$
f(t x+(1-t) y)+\frac{\rho}{2} \cdot t(1-t)\|x-y\|^{2} \leq t f(x)+(1-t) f(y)
$$

for all $x, y \in H$ and $t \in(0,1)$.

(v) $f$ is Gâteaux differentiable at $x \in H$ if there is $\nabla f(x) \in H$ such that

$$
\lim _{t \rightarrow 0} \frac{f(x+t y)-f(x)}{t}=\langle y, \nabla f(x)\rangle
$$

for each $y \in H$.

(vi) $f$ is Fréchet differentiable at $x$ if there is $\nabla f(x)$ such that

$$
\lim _{y \rightarrow 0} \frac{f(x+y)-f(x)-\langle\nabla f(x), y\rangle}{\|y\|}=0 .
$$

Definition 2.4. Let $f: H \rightarrow(-\infty, \infty]$ be a proper lower semicontinuous and convex function. Then the subdifferential $\partial f$ of $f$ is defined by

$$
\partial f(x):=\left\{x^{*} \in H: f(x)+\left\langle y-x, x^{*}\right\rangle \leq f(y) \text { for each } y \in H\right\}
$$

for each $x \in H$.

Lemma 2.1. [2, 4] Let $f: H \rightarrow(-\infty, \infty]$ be a proper lower semicontinuous and convex function. Then the following are satisfied. 
(i) $\partial f$ is a set-valued maximal monotone mapping.

(ii) $f$ is Gâteaux differentiable at $x \in \operatorname{int}(\mathscr{D})$ if and only if $\partial f(x)$ consists of a single element. That is, $\partial f(x)=\{\nabla f(x)\}$.

(iii) Suppose that $f$ is Fréchet differentiable. Then $f$ is convex if and only if $\nabla f$ is a monotone mapping.

Lemma 2.2. [2, Example 22.3(iv)] Let $\rho>0$ and let $H$ be a real Hilbert space. Let $f: H \rightarrow(\infty, \infty]$ be a proper lower-semicontinuous and convex function. If $f$ is $\rho$-strongly convex, then $\partial f$ is $\rho$-strongly monotone.

Lemma 2.3. [2, Proposition 16.26] Let $H$ be a real Hilbert space, and let $f: H \rightarrow(\infty, \infty]$ be a proper lower semicontinuous and convex function. If $\left\{u_{n}\right\}_{n \in \mathbb{N}}$ and $\left\{x_{n}\right\}_{n \in \mathbb{N}}$ are sequences in $H$ with $u_{n} \in \partial f\left(x_{n}\right)$ for all $n \in \mathbb{N}$, and $x_{n} \rightarrow x$ and $u_{n} \rightarrow u$, then $u \in \partial f(x)$.

\section{FORWARD-BACKWARD ALGORITHM FOR THE DCP}

Theorem 3.1. Let $\beta, \rho$, and $L$ be positive real numbers with $\beta \rho \geq 1 \geq \beta$ L. Let $\left\{k_{n}\right\}_{n \in \mathbb{N}}$ be a sequence of positive real numbers in $\left(0, \frac{3}{2}\right)$ with $\sum_{n=1}^{\infty} k_{n}\left(\frac{3}{2}-k_{n}\right)=\infty$. Let $H$ be a finite dimensional real Hilbert space. Let $g: H \rightarrow(-\infty, \infty]$ be a proper, $\rho$-strongly convex, and lower semicontinuous function, and let $h: H \rightarrow$ $(-\infty, \infty]$ be a Fréchet differentiable function with $\nabla h(x)$ is L-Lipschitz continuous. Let $f: H \rightarrow(-\infty, \infty]$ be defined by $f(x)=g(x)-h(x)$ for all $x \in H$ and assume that $f$ is bounded from below. Let $\Omega_{D C P}$ be defined by

$$
\Omega_{D C P}:=\{x \in H: \nabla h(x) \in \partial g(x)\},
$$

and assume that $\Omega_{D C P} \neq \emptyset$. Let $\left\{x_{n}\right\}_{n \in \mathbb{N}}$ be a sequence generated by

$$
\left\{\begin{array}{l}
x_{1} \in H, \\
z_{n}:=\arg \min _{v \in H}\left\{g(v)+\frac{1}{2 \beta}\left\|v-x_{n}\right\|^{2}-\left\langle\nabla h\left(x_{n}\right), v-x_{n}\right\rangle\right\}, \\
x_{n+1}:=x_{n}+k_{n}\left(z_{n}-x_{n}\right), n \in \mathbb{N} .
\end{array}\right.
$$

Then there exists $\bar{x} \in \Omega_{D C P}$ such that $\lim _{n \rightarrow \infty} x_{n}=\bar{x}$.

Proof. Take any $w \in \Omega_{\mathrm{DCP}}$ and let $w$ and $n$ be fixed. Note that

$$
\nabla h\left(x_{n}\right)+\frac{1}{\beta}\left(x_{n}-z_{n}\right) \in \partial g\left(z_{n}\right) .
$$

Since $g$ is $\rho$-strongly convex, we know $\partial g$ is $\rho$-strongly monotone. Hence, by (3.1), we have

$$
\rho\left\|z_{n}-w\right\|^{2} \leq\left\langle z_{n}-w, \nabla h\left(x_{n}\right)-\nabla h(w)+\frac{1}{\beta}\left(x_{n}-z_{n}\right)\right\rangle,
$$

which implies that

$$
\begin{aligned}
& 2 \beta \rho\left\|z_{n}-w\right\|^{2} \\
\leq & 2 \beta\left\langle z_{n}-w, \nabla h\left(x_{n}\right)-\nabla h(w)+\frac{1}{\beta}\left(x_{n}-z_{n}\right)\right\rangle \\
= & 2 \beta\left\langle z_{n}-w, \nabla h\left(x_{n}\right)-\nabla h(w)\right\rangle+2\left\langle z_{n}-w,\left(x_{n}-z_{n}+w\right)-w\right\rangle \\
\leq & 2 \beta L\left\|z_{n}-w\right\| \cdot\left\|x_{n}-w\right\|+2\left\langle z_{n}-w,\left(x_{n}-z_{n}+w\right)-w\right\rangle \\
\leq & \beta L\left\|z_{n}-w\right\|^{2}+\beta L\left\|x_{n}-w\right\|^{2}+\left\|z_{n}-w\right\|^{2}+\left\|x_{n}-z_{n}\right\|^{2} \\
& -\left\|2 z_{n}-x_{n}-w\right\|^{2} .
\end{aligned}
$$


Using (3.3), one has

$$
\left\|2 z_{n}-x_{n}-w\right\|^{2} \leq(1+\beta L-2 \beta \rho)\left\|z_{n}-w\right\|^{2}+\beta L\left\|x_{n}-w\right\|^{2}+\left\|x_{n}-z_{n}\right\|^{2} .
$$

It follows that

$$
\begin{aligned}
& \left\|x_{n+1}-w\right\|^{2} \\
= & \left\|x_{n}+k_{n}\left(z_{n}-x_{n}\right)-w\right\|^{2} \\
= & \left\|\left(1-\frac{k_{n}}{2}\right)\left(x_{n}-w\right)+\frac{k_{n}}{2}\left(2 z_{n}-x_{n}-w\right)\right\|^{2} \\
= & \left(1-\frac{k_{n}}{2}\right)\left\|x_{n}-w\right\|^{2}+\frac{k_{n}}{2}\left\|2 z_{n}-x_{n}-w\right\|^{2} \\
& -\frac{k_{n}}{2} \cdot\left(1-\frac{k_{n}}{2}\right)\left\|\left(2 z_{n}-x_{n}-w\right)-\left(x_{n}-w\right)\right\|^{2} \\
\leq & \left(1-\frac{k_{n}}{2}\right)\left\|x_{n}-w\right\|^{2}+\frac{k_{n}}{2}\left\|2 z_{n}-x_{n}-w\right\|^{2}-k_{n}\left(2-k_{n}\right)\left\|z_{n}-x_{n}\right\|^{2} \\
\leq & \left(1-\frac{k_{n}}{2}\right)\left\|x_{n}-w\right\|^{2}-k_{n}\left(2-k_{n}\right)\left\|z_{n}-x_{n}\right\|^{2} \\
& +\frac{k_{n}}{2}\left[(1+\beta L-2 \beta \rho)\left\|z_{n}-w\right\|^{2}+\beta L\left\|x_{n}-w\right\|^{2}+\left\|x_{n}-z_{n}\right\|^{2}\right] \\
\leq & \left\|x_{n}-w\right\|\left\|^{2}-k_{n}\left(\frac{3}{2}-k_{n}\right)\right\| x_{n}-z_{n} \|^{2} .
\end{aligned}
$$

In view of (3.5), we find that $\lim _{n \rightarrow \infty}|| x_{n}-w \|$ exists, and $\lim _{n \rightarrow \infty}|| x_{n}-z_{n} \|=0$. Further, $\left\{x_{n}\right\}_{n \in \mathbb{N}}$ and $\left\{z_{n}\right\}_{n \in \mathbb{N}}$ are bounded sequences. So, we may assume that there exist subsequences $\left\{x_{n_{j}}\right\}_{j \in \mathbb{N}}$ and $\left\{z_{n_{j}}\right\}_{j \in \mathbb{N}}$ of $\left\{x_{n}\right\}_{n \in \mathbb{N}}$ and $\left\{z_{n}\right\}_{n \in \mathbb{N}}$, respectively, such that $x_{n_{j}} \rightarrow \bar{x}$ and $z_{n_{j}} \rightarrow \bar{x}$ for some $\bar{x} \in H$. It follows from (3.1) and Lemma 2.3 that $\nabla h(\bar{x}) \in \partial g(\bar{x})$, that is, $\bar{x} \in \Omega_{\mathrm{DCP}}$. Hence, $\lim _{n \rightarrow \infty}\left\|x_{n}-\bar{x}\right\|$ exists and

$$
\lim _{n \rightarrow \infty}|| x_{n}-\bar{x}||=\lim _{j \rightarrow \infty}\left\|x_{n_{j}}-\bar{x}\right\|=0 .
$$

This completes the proof.

Remark 3.1. For the condition $\beta \rho \geq 1 \geq \beta L$ in Theorem 3.1, we know that $\rho \geq L$. Thus,

(a) if $\rho=L$, then we set $\beta=\frac{1}{L}$;

(b) if $\rho>L$, then we may choose $\beta \in\left[\frac{1}{\rho}, \frac{1}{L}\right]$.

Besides, we know the conclusion of Theorem 3.1 is still true if we assume that $\liminf _{n \rightarrow \infty} k_{n}\left(\frac{3}{2}-k_{n}\right)>0$. If $k_{n}=1$, then Theorem 3.1 is reduced to be the following convergence theorem.

Theorem 3.2. [18] Let $\beta$, $\rho$, and $L$ be positive real numbers with $\beta \rho \geq 1 \geq \beta L$. Let $H$ be a finite dimensional real Hilbert space. Let $g: H \rightarrow(-\infty, \infty]$ be a proper, $\rho$-strongly convex, and lower semicontinuous function, and let $h: H \rightarrow(-\infty, \infty]$ be a Fréchet differentiable function with $\nabla h(x)$ is L-Lipschitz continuous. Let $f: H \rightarrow(-\infty, \infty]$ be defined by $f(x)=g(x)-h(x)$ for all $x \in H$, and assume that $f$ is bounded from below. Let $\Omega_{D C P}$ be defined by $\Omega_{D C P}:=\{x \in H: \nabla h(x) \in \partial g(x)\}$, and assume that $\Omega_{D C P} \neq \emptyset$. Let $\left\{x_{n}\right\}_{n \in \mathbb{N}}$ be a sequence generated by

$$
x_{1} \in H, \quad x_{n+1}:=\arg \min _{v \in H}\left\{g(v)+\frac{1}{2 \beta}\left\|v-x_{n}\right\|^{2}-\left\langle\nabla h\left(x_{n}\right), v-x_{n}\right\rangle\right\}, n \in \mathbb{N} .
$$

Then there exists $\bar{x} \in \Omega_{D C P}$ such that $\lim _{n \rightarrow \infty} x_{n}=\bar{x}$.

To give the next convergence theorem, we need the following result presented by Beck and Teboulle [3]. 
Lemma 3.1. [3, Lemma 4.3] Let $t_{0}=1$ and $\left\{t_{n}\right\}_{n \in \mathbb{N}}$ be generated by

$$
t_{n+1}:=\frac{1+\sqrt{1+4 t_{n}^{2}}}{2} .
$$

Then $\frac{n+1}{2} \leq t_{n}$ for all $n \in \mathbb{N}$.

The following is a convergence theorem for DC programming using Beck-Teboule proximal gradient algorithm.

Theorem 3.3. Let $\beta, \rho$, and $L$ be positive real numbers with $\beta \rho \geq 1 \geq \beta$ L. Let $H$ be a finite dimensional real Hilbert space. Let $g: H \rightarrow(-\infty, \infty]$ be a proper, $\rho$-strongly convex, and lower semicontinuous function, and let $h: H \rightarrow(-\infty, \infty]$ be a Fréchet differentiable function with $\nabla h(x)$ is L-Lipschitz continuous. Let $f: H \rightarrow(-\infty, \infty]$ be defined by $f(x)=g(x)-h(x)$ for all $x \in H$, and assume that $f$ is bounded from below. Let $\Omega_{D C P}$ be defined by $\Omega_{D C P}:=\{x \in H: \nabla h(x) \in \partial g(x)\}$, and assume that $\Omega_{D C P} \neq \emptyset$. Let $x_{1} \in H, t_{0}=1$ and $\left\{x_{n}\right\}_{n \in \mathbb{N}}$ and $\left\{t_{n}\right\}_{n \in \mathbb{N}}$ be generated by

$$
\left\{\begin{aligned}
z_{n} & :=\arg \min _{v \in H}\left\{g(v)+\frac{1}{2 \beta}\left\|v-x_{n}\right\|^{2}-\left\langle\nabla h\left(x_{n}\right), v-x_{n}\right\rangle\right\}, \\
t_{n+1} & :=\frac{1+\sqrt{1+4 t_{n}^{2}}}{2} \\
x_{n+1} & :=x_{n}+\frac{t_{n}-1}{t_{n+1}}\left(z_{n}-x_{n}\right), n \in \mathbb{N} .
\end{aligned}\right.
$$

Then $\left\{x_{n}\right\}_{n \in \mathbb{N}}$ converges to an element of $\Omega_{D C P}$.

Proof. For each $n \in \mathbb{N}$, let $k_{n}$ be defined by

$$
k_{n}=\frac{t_{n}-1}{t_{n+1}}\left(\frac{3}{2}-\frac{t_{n}-1}{t_{n+1}}\right), n \in \mathbb{N} .
$$

Since $\frac{n+1}{2} \leq t_{n}$, we know $t_{n} \geq 1$ and

$$
t_{n+1}=\frac{1+\sqrt{1+4 t_{n}^{2}}}{2} \leq \frac{1+\sqrt{4 t_{n}^{2}+2 t_{n}-n}}{2}<\frac{1+2 t_{n}+0.5}{2}=t_{n}+\frac{3}{4}, n \in \mathbb{N} .
$$

By induction method, we know

Hence,

$$
t_{n}<1+\frac{3 n}{4}<1+n, n \in \mathbb{N}
$$

which implies that

$$
\frac{n-1}{2} \leq t_{n}-1 \leq \frac{3 n}{4}
$$

$$
0 \leq \frac{n-1}{2(n+2)} \leq \frac{t_{n}-1}{t_{n+1}} \leq \frac{3 n}{4} \cdot \frac{2}{n+2}=\frac{3 n}{2(n+2)}<\frac{3}{2}, n \in \mathbb{N}
$$

Further, we have

$$
\frac{t_{n}-1}{t_{n+1}}\left(\frac{3}{2}-\frac{t_{n}-1}{t_{n+1}}\right) \geq \frac{n-1}{2(n+2)} \cdot\left(\frac{3}{2}-\frac{3 n}{2(n+2)}\right)=\frac{3(n-1)}{2(n+2)^{2}}
$$

Therefore, we obtain

$$
\sum_{n=1}^{\infty} \frac{t_{n}-1}{t_{n+1}}\left(\frac{3}{2}-\frac{t_{n}-1}{t_{n+1}}\right)=\infty .
$$

Therefore, we conclude the proof of Theorem 3.3 from Theorem 3.2. 
Theorem 3.4. Let $\beta$, $\rho$, and $L$ be positive real numbers with $\beta \rho \geq 1 \geq \beta$ L. Let $\left\{t_{n}\right\}_{n \in \mathbb{N}}$ be a sequence of positive real numbers in $\left(-\frac{1}{2}, \frac{1}{2}\right)$ with $\sum_{n=1}^{\infty}\left[\left(1+t_{n}\right)\left(1-2 t_{n}\right)\right]=\infty$. Let $H$ be a finite dimensional real Hilbert space. Let $g: H \rightarrow(-\infty, \infty]$ be a proper, $\rho$-strongly convex, and lower semicontinuous function, and let $h: H \rightarrow(-\infty, \infty]$ be a Fréchet differentiable function with $\nabla h(x)$ is L-Lipschitz continuous. Let $f: H \rightarrow(-\infty, \infty]$ be defined by $f(x)=g(x)-h(x)$ for all $x \in H$, and assume that $f$ is bounded from below. Let $\Omega_{D C P}$ be defined by $\Omega_{D C P}:=\{x \in H: \nabla h(x) \in \partial g(x)\}$, and assume that $\Omega_{D C P} \neq \emptyset$. Let $\left\{x_{n}\right\}_{n \in \mathbb{N}}$ be a sequence generated by

$$
\left\{\begin{array}{l}
x_{1} \in H \\
z_{n}:=\arg \min _{v \in H}\left\{g(v)+\frac{1}{2 \beta}\left\|v-x_{n}\right\|^{2}-\left\langle\nabla h\left(x_{n}\right), v-x_{n}\right\rangle\right\} \\
x_{n+1}:=z_{n}+t_{n}\left(z_{n}-x_{n}\right), n \in \mathbb{N} .
\end{array}\right.
$$

Then there exists $\bar{x} \in \Omega_{D C P}$ such that $\lim _{n \rightarrow \infty} x_{n}=\bar{x}$.

Proof. Let $k_{n}=1+t_{n}$. From Theorem 3.1, we can obtain our desired conclusion immediately.

Further, we give the following modified forward-backward algorithm for DC programming.

Theorem 3.5. Let $\beta$, $\rho$, and $L$ be positive real numbers with $\beta \rho \geq 1 \geq \beta$ L. Let $\left\{k_{n}\right\}_{n \in \mathbb{N}}$ be a sequence of positive real numbers in $\left(0, \frac{3}{2}\right)$ with $\sum_{n=1}^{\infty} k_{n}\left(\frac{3}{2}-k_{n}\right)=\infty$. Let $H$ be a finite dimensional real Hilbert space. Let $g: H \rightarrow(-\infty, \infty]$ be a proper, $\rho$-strongly convex, and lower semicontinuous function, and let $h: H \rightarrow$ $(-\infty, \infty]$ be a Fréchet differentiable function with $\nabla h(x)$ is L-Lipschitz continuous. Let $f: H \rightarrow(-\infty, \infty]$ be defined by $f(x)=g(x)-h(x)$ for all $x \in H$, and assume that $f$ is bounded from below. Let $\Omega_{D C P}$ be defined by

$$
\Omega_{D C P}:=\{x \in H: \nabla h(x) \in \partial g(x)\},
$$

and assume that $\Omega_{D C P} \neq \emptyset$. Let $\left\{x_{n}\right\}_{n \in \mathbb{N}}$ be a sequence generated by

$$
\left\{\begin{array}{l}
x_{1} \in H \\
z_{n}:=\arg \min _{v \in H}\left\{g(v)+\frac{1}{2 \beta}\left\|v-x_{n}\right\|^{2}-\left\langle\nabla h\left(x_{n}\right), v-x_{n}\right\rangle\right\} \\
x_{n+1}:=z_{n}+k_{n}\left(z_{n}-z_{n-1}\right), n \in \mathbb{N} .
\end{array}\right.
$$

Then there exists $\bar{x} \in \Omega_{D C P}$ such that $\lim _{n \rightarrow \infty} x_{n}=\bar{x}$.

Next, we give convergence theorems for the generalized DC programming.

Theorem 3.6. Let $\beta, \rho$, and $L$ be positive real numbers with $2 \beta \rho \geq 2>1 \geq \beta$ L. Let $\left\{k_{n}\right\}_{n \in \mathbb{N}}$ be a sequence of positive real numbers in $\left(0, \frac{3}{2}\right)$ with $\sum_{n=1}^{\infty} k_{n}\left(\frac{3}{2}-k_{n}\right)=\infty$. Let $H$ be a finite dimensional real Hilbert space. Let $g: H \rightarrow(-\infty, \infty]$ be a proper, $\rho$-strongly convex, and lower semicontinuous function, and let $h, \varphi: H \rightarrow(-\infty, \infty]$ be Fréchet differentiable functions with $\nabla h(x)$ and $\nabla \varphi(x)$ are L-Lipschitz continuous. Let $f: H \rightarrow(-\infty, \infty]$ be defined by $f(x)=\varphi(x)+g(x)-h(x)$ for all $x \in H$, and assume that $f$ is bounded from below. Let $\Omega_{G D C P}$ be defined by

$$
\Omega_{G D C P}:=\{x \in H: \nabla h(x)-\nabla \varphi(x) \in \partial g(x)\},
$$


and assume that $\Omega_{G D C P} \neq \emptyset$. Let $\left\{x_{n}\right\}_{n \in \mathbb{N}}$ be a sequence generated by

$$
\left\{\begin{array}{l}
x_{1} \in H, \\
z_{n}:=\arg \min _{v \in H}\left\{g(v)+\frac{1}{2 \beta}\left\|v-x_{n}\right\|^{2}-\left\langle\nabla h\left(x_{n}\right)-\nabla \varphi\left(x_{n}\right), v-x_{n}\right\rangle\right\}, \\
x_{n+1}:=x_{n}+k_{n}\left(z_{n}-x_{n}\right), n \in \mathbb{N} .
\end{array}\right.
$$

Then there exists $\bar{x} \in \Omega_{G D C P}$ such that $\lim _{n \rightarrow \infty} x_{n}=\bar{x}$.

Proof. Following the same argument as the proof of Theorem 3.1, we conclude the conclusion of Theorem 3.6 immediately.

Theorem 3.7. Let $\beta, \rho$, and $L$ be positive real numbers with $2 \beta \rho \geq 2>1 \geq \beta L$. Let $H$ be a finite dimensional real Hilbert space. Let $g: H \rightarrow(-\infty, \infty]$ be a proper, $\rho$-strongly convex, and lower semicontinuous function, and let $h, \varphi: H \rightarrow(-\infty, \infty]$ be Fréchet differentiable functions with $\nabla h(x)$ and $\nabla \varphi(x)$ are L-Lipschitz continuous. Let $f: H \rightarrow(-\infty, \infty]$ be defined by $f(x)=\varphi(x)+g(x)-h(x)$ for all $x \in H$, and assume that $f$ is bounded from below. Let $\Omega_{G D C P}$ be defined by

$$
\Omega_{G D C P}:=\{x \in H: \nabla h(x)-\nabla \varphi(x) \in \partial g(x)\},
$$

and assume that $\Omega_{G D C P} \neq \emptyset$. Let $\left\{x_{n}\right\}_{n \in \mathbb{N}}$ be a sequence generated by

$$
\left\{\begin{array}{l}
x_{1} \in H \\
z_{n}:=\arg \min _{v \in H}\left\{g(v)+\frac{1}{2 \beta}\left\|v-x_{n}\right\|^{2}-\left\langle\nabla h\left(x_{n}\right)-\nabla \varphi\left(x_{n}\right), v-x_{n}\right\rangle\right\} \\
t_{n+1}:=\frac{1+\sqrt{1+4 t_{n}^{2}}}{2} \\
x_{n+1}:=x_{n}+\frac{t_{n}-1}{t_{n+1}}\left(z_{n}-x_{n}\right), n \in \mathbb{N} .
\end{array}\right.
$$

Then there exists $\bar{x} \in \Omega_{G D C P}$ such that $\lim _{n \rightarrow \infty} x_{n}=\bar{x}$.

Proof. From Theorem 3.3 and Theorem 3.6, we obtain the conclusion of Theorem 3.7 immediately.

\section{REFERENCES}

[1] N.T. An, N.M. Nam, Convergence analysis of a proximal point algorithm for minimizing differences of functions, Optimization, 66 (2017), 129-147.

[2] H.H. Bauschke, P.L. Combettes, Convex Analysis and Monotone Operator Theory in Hilbert Spaces, Springer, New York, 2011.

[3] A. Beck, M. Teboulle, A fast iterative shrinkage-thresholding algorithm for linear inverse problems, SIAM J. Image. Sci. 2 (2009), 183-202.

[4] D. Butnariu, A.N. Iusem, Totally Convex Functions for Fixed Points Computation and Infinite Dimensional Optimization, Kluwer Academic Publishers, The Netherlands, 2000.

[5] P.L. Combettes, V.R. Wajs, Signal recovery by proximal forward-backward splitting, Multiscale Model. Simul. 4 (2005), 1168-1200.

[6] B.V. Dinh, D.S. Kim, L. Jiao, Convergence analysis of algorithms for DC programming, arXiv:1508.03899v1 [math.OC], (2015).

[7] D.H. Fang, X. Gong, Extended Farkas lemma and strong duality for composite optimization problems with DC functions, Optimization, 66 (2017), 179-196.

[8] D.H. Fang, G.M. Lee, C. Li, J.C. Yao, Extended Farkas's lemmas and strong Lagrange dualities for DC infinite programming, J. Nonlinear Convex Anal. 14 (2013), 747-767. 
[9] D.H. Fang, C. Li, X.Q. Yang, Stable and total fenchel duality for DC optimization problems in locally convex spaces, SIAM J. Optim. 21 (2011), 730-760.

[10] D.H. Fang, X.P. Zhao, Local and global optimality conditions for DC infinite optimization problems, Taiwanese J. Math. 18 (2014), 817-834.

[11] D.H. Fang, X.P. Zhao, Optimality conditions for convex and DC infinite optimization problems, J. Nonlinear Convex Anal. 17 (2016), 683-700.

[12] Y. Fujikara, D. Kuroiwa, Lagrange duality in canonical DC programming, J. Math. Anal. Appl 408 (2013), $476-483$.

[13] R. Harada, D. Kuroiwa, Lagrange-type in DC programming, J. Math. Anal. Appl. 418 (2014), 415-424.

[14] J.B. Hiriart-Urruty, H. Tuy, Essays on nonconvex optimization, Math. Program. Vol. 41, North-Holland, 1988.

[15] A.J. Levy, A fast quadratic programming algorithm for positive signal restoration, IEEE Trans. Acoust. Speech Signal Process 31 (1983), 1337-1341.

[16] P. E. Maingé, A. Moudafi, Convergence of new inertial proximal methods for DC programming, SIAM J. Optim. 19 (2008), 397-413.

[17] Y. Saeki, D. Kuroiwa, Optimality conditions for DC programming problems with reverse convex constraints, Nonlinear Anal. 80 (2013), 18-27.

[18] J.C.O. Souza, P.R. Oliveira, A. Soubeyran, Global convergence of a proximal linearized algorithm for difference of convex functions, Optim. Lett. 10 (2016), 1529-1539.

[19] W. Sun, R.J.B. Sampaio, M.A.B. Candido, Proximal point algorithm for minimization of DC functions, J. Comput. Math. 21 (2003), 451-462. 